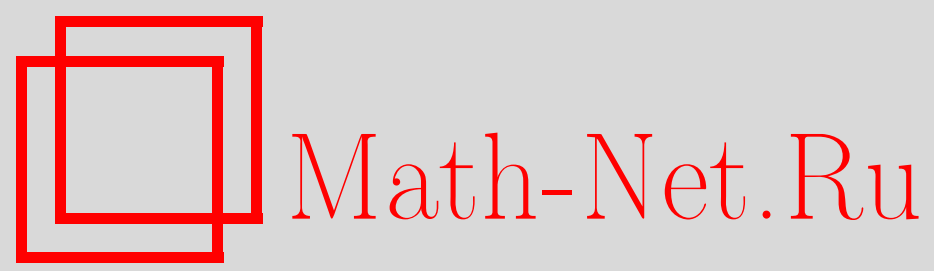

Л. В. Кудюров, С. Л. Кудюров, В. В. Митин, Математическое обоснование механизма равномерного перемещения аудиторной доски, Вестн. Сам. гос. техн. ун-та. Сер. Физ.-мат. науки, 2002, выпуск 16, 211-212

DOI: https://doi.org/10.14498/vsgtu128

Использование Общероссийского математического портала Math-Net.Ru подразумевает, что вы прочитали и согласны с пользовательским соглашением

http: //www.mathnet.ru/rus/agreement

Параметры загрузки:

IP : 54.166 .219 .16

26 апреля 2023 г., $15: 48: 30$ 


\section{МАТЕМАТИЧЕСКОЕ ОБОСНОВАНИЕ МЕХАНИЗМА РАВНОМЕРНОГО ПЕРЕМЕЩЕНИЯ АУДИТОРНОЙ ДОСКИ}

Дано математическое обоснование механизма перемещения аудиторной доски с барабаном, рабочий профиль которого выполнен в виде логарифмической спирали, что обеспечивает равномерный подъём или опускание объекта.

Рассматривается механизм подвески аудиторной доски (см. рисунок), обеспечивающий ее плавное перемещение в вертикальной плоскости. Механизм включает систему пружин 1 , прикрепленных в точке $\mathrm{O}_{1}$ к доске, а в точке $\mathrm{C}$ - к тросику 2 , который другим концом прикреплен к точке М барабана 3 и может вращаться вокруг оси, проходящей через точку О доски. К барабану прикреплены тросики 4 и 5, которые перекинуты через ролики 6 и 7 подвески и прикреплены другими своими концами в точках L и К к стенке. Длина тросиков такова, что они постоянно находятся в натянутом состоянии, обеспечивая фиксацию доски на пути перемещения. Когда доска находится в крайнем верхнем положении, правый конец тросика 2 находится в положении $\mathrm{M}$, а его левый конец $\mathrm{C}$ - в положении $\mathrm{O}_{2}$. При этом пружина вытянута на величину $\lambda$. При перемещении доски вниз тросик 2 наворачивается на дугу MN рабочего профиля барабана, которая занимает положение $\mathrm{M}_{2} \mathrm{~N}_{2}$ в крайнем нижнем положении доски. В отличие от известных методов для обеспечения плавного движения доски в любой момент времени тросик 2 перекинут через систему двух смежных роликов 6, что позволяет тросику 2 постоянно сходить по касательной рабочему профилю MN. Покажем, что такая система обеспечивает равномерное движение доски по направляющим. Следует отметить, что доска, будучи остановлена на любой возможной высоте, должна оставаться в покое, если к ней не приложить небольшое усилие. Чтобы это обеспечить, нужно постоянно выполнять условие равновесия моментов сил относительно точки О, т.е.

$$
F_{B} \cdot \rho \sin \alpha=P \cdot R=\text { const },
$$

где - $F_{6}$ - восстанавливающая сила, $\rho$ - радиус рабочего профиля, $\alpha$ - угол между $\rho$ и касательной к кривой профиля, $P$ - сила тяжести объекта, $R$ - радиус барабана.

Но $F_{6}$ есть функция деформации пружины $s$, т. е.

$$
F_{6}=c_{\ni}(\lambda+s),
$$

где $c_{\ni}$ - эквивалентная жесткость; $\lambda$ - статическая деформация, $s$ - текущая деформация пружины, отсчитываемая от положения статического равновесия. При этом $F_{6}$ и $\rho \sin \alpha$ должны изменяться обратно пропорционально согласно (1), в силу чего профиль рабочей поверхности барабана должен быть специальной формы, обеспечивающей (1). Для равномерного изменения $s$ необходимо, чтобы угол $\alpha$ был постоянным, поэтому форма профиля барабана выполнена в виде логарифмической спирали:

$$
\rho=R e^{-k \varphi},
$$

где $\mathrm{k}=\operatorname{ctg} \alpha ; \varphi-$ угол поворота барабана. Логарифмическая спираль обладает одним замечательным свойством, заключающимся в том, что угол $\alpha$ между радиусом спирали $\rho$ и касательной в любой точке кривой один и тот же (этот факт облегчает технологию изготовления такого профиля).

Так как $d s \sin \alpha=\rho d \varphi=R e^{-k \varphi}$, то интегрируя с учетом граничных условий ( $\left.s=0, \varphi=0, s=L_{s}, \varphi=\varphi_{s}\right)$, получим длину профиля в виде

$$
L_{s}=\frac{R\left(1-e^{-k \varphi_{s}}\right)}{\cos \alpha},
$$

где $\varphi_{s}$ - угол, стягиваемый дугой $\mathrm{L}_{\mathrm{s}}$. Пренебрегая силами трения и полагая, что скорость перемещения объекта постоянна, получим

$$
F_{B}^{\prime} \cdot d s+\stackrel{r}{P} \cdot d h=0
$$

Здесь $d s^{\prime}$ и $d h^{\prime}-$ векторы элементарных перемещений. Из решения (4) с учетом граничных условий (при $\varphi=\varphi_{s} h=H, s=L_{s}$ ) следует, что 


$$
L_{s}=-\lambda+\sqrt{\left(\lambda^{2}+2 P H / c_{\ni}\right.},
$$

где $H$-заданное максимальное перемещение объекта.

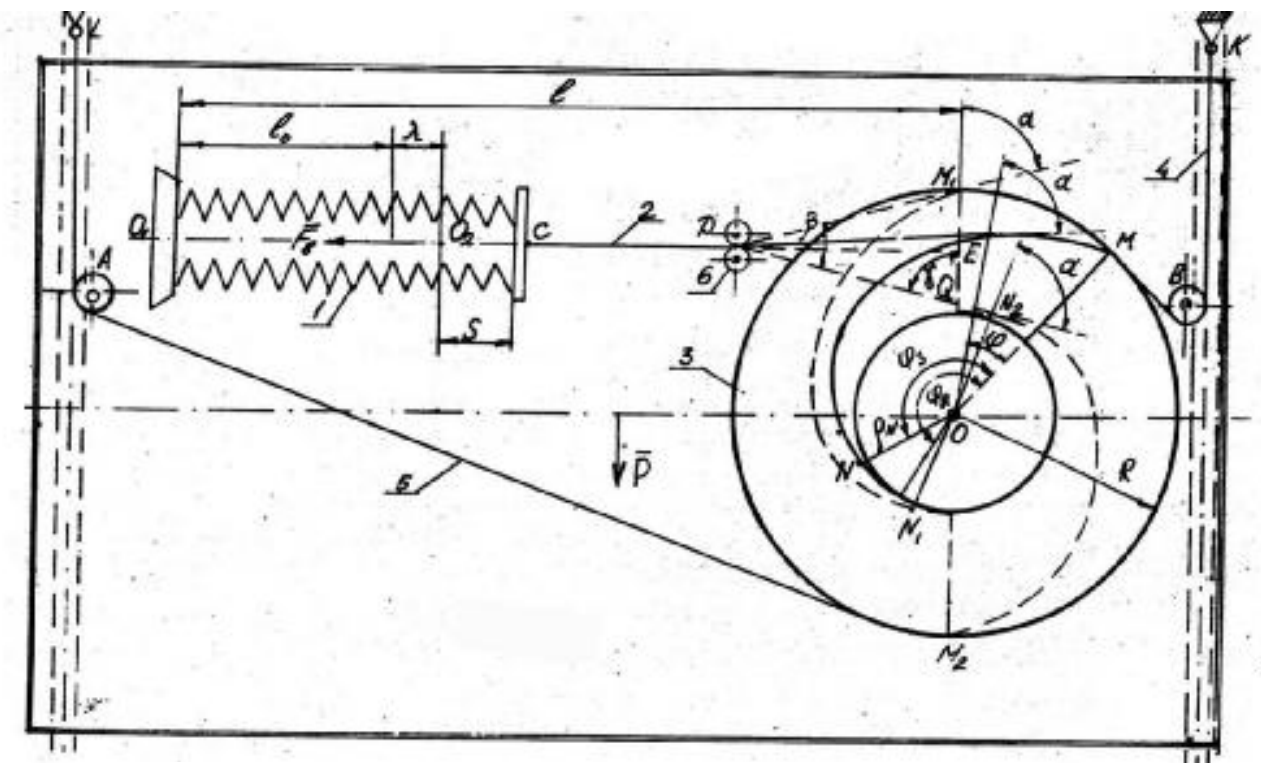

Механизм перемещения аудиторной доски

Сравнивая (3) и (5), найдем радиус барабана:

$$
R=\left(-\lambda+\sqrt{\lambda^{2}+2 P H / c_{\ni}}\right) /\left(1-e^{-k \varphi_{s}}\right) .
$$

Из геометрических соображений следует

$$
\varphi_{s}=H / R+2 \alpha-\pi, \quad D M_{1}=M_{1} Q \sin \gamma / \sin \beta,
$$

где $\gamma=\pi-\left(H / R-\varphi_{S}\right)-\alpha, \beta=\left(H / R-\varphi_{s}\right), D E=D M_{1} \sin \alpha$. Длина невытянутой пружины $l_{0}=l-\lambda-L_{S}-D E$, где $l$ - базовый размер механизма. Как показывают численные расчеты, жесткость пружины сильно зависит от величины угла $\alpha$. С ростом $\alpha$ эквивалентная жесткость быстро уменьшается.

\section{УДК 536.24}

\section{А.А. Базаров}

\section{СИНТЕЗ ЗАМКНУТОЙ СИСТЕМЫ УПРАВЛЕНИЯ ДЛЯ ОБЪЕКТА С РАСПРЕДЕЛЕННЫМИ ПАРАМЕТРАМИ}

Рассмотрена процедура построения алгоритма решения оптимальной задачи для системы с распределенными параметрами. Предложен способ, базирующийся на комбинаџии теории синтеза оптимального управления для систем с сосредоточенными параметрами и метода конечноэлементных элементов.

Для синтеза замкнутых систем управления для объектов с распределенными параметрами (СРП) используются два подхода: аппроксимация системой с сосредоточенными параметрами (ССП) и применение аппарата синтеза систем управления для ССП; применение аппарата синтеза для СРП. Первый способ является более разработанным, но имеет такие недостатки, как упрощение системы и утрата некоторой части информации. Второй способ имеет более сложную теорию и требует весьма значительных вычислительных ресурсов, но позволяет получать более точные результаты. Среди множества методов синтеза для СРП все большее распространение приобретают численные методы, отличающиеся многообразием поисковых процедур, но малонаглядные. Вместе с тем первый способ обладает хорошо формализуемым и наглядным алгоритмом синтеза. С учетом изложенного предлагается методика синтеза, сочетающая преимущества обоих подходов. 